\title{
A Study on Person Verification Using Electroencephalograms Evoked by Unperceivable Vibration Stimuli
}

\author{
$1^{\text {st }}$ Yoshiaki Shindo \\ Graduate School of Sustainable Sciences \\ Tottori University \\ Tottori, Japan \\ m18j4028b@edu.tottori-u.ac.jp
}

\author{
$2^{\text {nd }}$ Isao Nakanishi \\ Faculty of Engineering \\ Tottori University \\ Tottori, Japan \\ nakanishi@ tottori-u.ac.jp
}

\author{
$3^{\text {rd }}$ Akinobu Takemura \\ Graduate School of Engineering \\ Tottori University \\ Tottori, Japan \\ b12t3035@faraday.ele.tottori-u.ac.jp
}

\begin{abstract}
In this study, the person verification using brainwaves evoked by unperceivable vibration stimulation is proposed. Brainwaves in the participants were measured while they were given unperceivable vibration stimuli, and their inducibility and individuality were examined on the basis of a $t$-test using spectral content rates in $\theta, \alpha$, and $\beta$ wavebands. In addition, the verification performance using the content rates as an individual feature was evaluated using Euclidian distance matching. The average equal error rate of all electrodes was approximately 34 $\%$.
\end{abstract}

Index Terms-biometrics, brain wave, tactile stimulation, unperceivable vibration, $t$-test

\section{INTRODUCTION}

Biometric technology, such as fingerprints and facial recognition has been introduced in recent years and is now commonly used in devices such as smartphones. However, biometric data invariably involve body surfaces, making theft easy; for instance, facial images can easily be captured on a digital camera and copies can be made. In addition, fingerprint and facial recognition assume one-time-only authentication, so there is a risk of spoofing. After verification by a regular user of a system using his/her biometric data, even if he/she is switched to an imposter who does not have a license to use the system, it is impossible to detect spoofing using biometrics based on one-time-only authentication.

In order to deal with this problem, continuous authentication is more effective than one-time-only authentication. As biometrics suitable for continuous authentication, brainwaves (observed using an electroencephalogram, EEG) have attracted a significant deal of attention [1]. Using EEGs evoked by a stimulus is far more effective than using spontaneous ones. However, that stimulus must be unperceivable by human beings. If a perceivable stimulus is given to a user while he/she is using a system, it disturbs system usage.

Nakanishi et al. proposed using an image in a moving picture with a high frame rate as an invisible stimulus [2] and ultrasound as an inaudible stimulus [3]. In this study, the authors measured the EEGs in the participants while they were given unperceivable vibration stimuli and examined the inducibility and individuality of the measured EEGs on the basis of a $t$-test using spectral content ratios in $\theta, \alpha$, and $\beta$ wavebands. In addition, the verification performance of the EEGs using the content ratios as an individual feature was evaluated on the basis of Euclidian distance matching.

\section{Person Verification Using EeGs Evoked by UNPERCEIVABLE VIBRATION STIMULI}

The use of brainwave as biometrics has previously been studied [1]. Electrical time variations from a large number of synapses (neurons) in the cerebral cortex are accumulated and then detected as a brainwave on the scalp using an electrode. In other words, brainwaves are never detected without placing an electrode on the scalp; therefore, this method is more secure than other conventional biometrics. Once the electrodes are placed on the scalp of the user, the user is no longer conscious of them. This is suitable for continuous authentication.

Brainwaves are categorized as either spontaneous or evoked. Spontaneous brainwaves always occur independently of a specified event. Person authentication using spontaneous brainwaves has been widely studied, and the use of evoked responses in brainwaves as biometrics has also proposed [4]. Evoked brainwaves are those that result from an endogenous or exogenous stimulus. If there are more individualities in the induced brainwaves, they are expected to obtain a higher verification performance compared to those use spontaneous brainwaves.

In this study, the authors assume continuous system user authentication. In this case, the stimulation must be unperceivable to the users since a perceivable stimulus disturbs their continuous use of the system. Unperceivable stimulation is defined as a stimulation that is not perceivable but is presented practically to the users. Any responses are detected by the sensory organs and then transmitted to the brain. Person authentication using unperceivable visual and audible stimuli has already been proposed, and it has been confirmed that responses to these stimuli are different from those received by perceivable stimulation [2], [3]. 


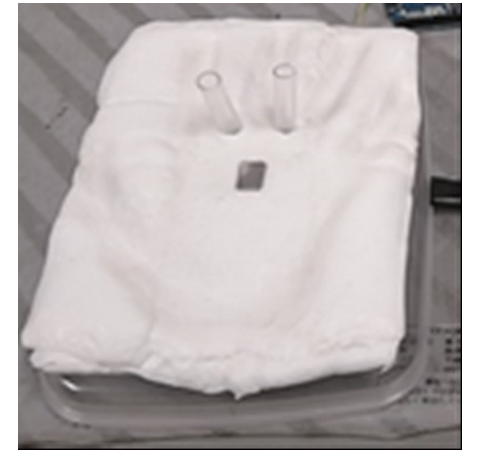

Fig. 1. A device for presenting a vibrational stimulation.

The authors of this study propose using unperceivable tactile stimulation. In the case of using visible and audible stimulation, there is a space between the origin of the stimulus (a display or a speaker) and the user, and this influences their perception. On the other hand, tactile stimulation is presented directly to the skin; therefore, a direct and stable response in brainwaves is expected. However, no research on brainwaves evoked by unperceivable tactile stimulation has been undertaken. Before evaluating the authentication performance, it is necessary to confirm whether any response is evoked by unperceivable tactile stimulation.

In general, "tactile" refers to cutaneous (skin) sensation such as touch, pressure, pain, and temperature. Cutaneous receptors in the skin feel these sensation and then yield senses. Tactile stimulation is a collective term for pressure, temperature, vibration, and electrical stimulation. In this study, the authors used a vibration to stimulate the palm using a vibrational actuator.

Figure 1 shows a device for applying tactile stimulation, in which a vibrational stimulus is generated by a vibrational actuator (square one in Fig. 1) and two transparent acrylic poles are the guide for fixing the actuator position on a palm. A palm is put on the device and a long finger is placed between the two poles, with the interdigital areas of both sides of the finger touching the poles. The drive voltage of the actuator used in this study was $5 \mathrm{~V}$ and the pulse width was $200 \mu$ s. The interval between pulses can be controlled as a vibration cycle (frequency). The stimulation was very weak; therefore, it is assumed to have little influence on the human body. However, the degree of influence is an area for future research.

There are four sensory receptors, Merkel cells, Ruffini corpuscles, Meissner corpuscles and Pacinian corpuscles, with different sensation thresholds of vibration frequency [5]. The authors, therefore, prepared unperceivable vibration stimuli whose frequencies were a little higher than these thresholds.

First, the authors investigated the sensation thresholds in the participants. There were 20 participants, all of whom were males. The number of measured data per participant was 15. While increasing the vibration frequency by $10 \mathrm{~Hz}$, the participant were required to show some response when they sensed the vibration. Figure 2 shows the sensed frequency bands of all the participants. The sensation threshold of each

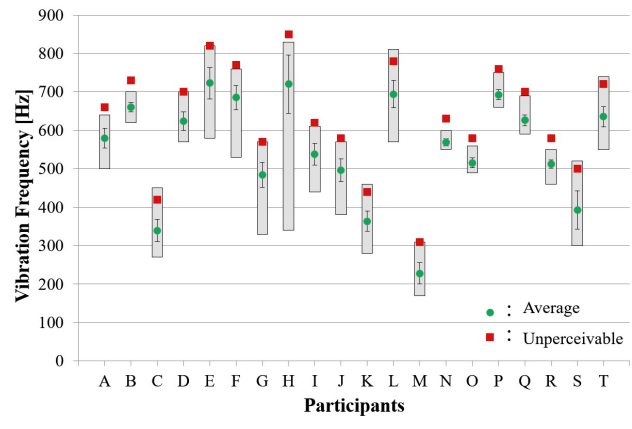

Fig. 2. Sensed frequency bands of all participants.

participant differed because the thickness and hardness of skin in each individual were different [6].

Second, the authors statistically calculated a $95 \%$ confidence interval among the sensed frequencies of each participant. The confidence intervals are indicated as vertical bars in Fig. 2.

Finally, the authors added $50 \mathrm{~Hz}$ to the upper limit of the 95\% confidence interval, which resulted in a personalized vibration frequency for each individual; this is indicated as a red box in Fig. 2. It is assumed that a vibration stimulus with such a frequency is not perceived by a participant but is at least received by his cutaneous receptors, evoking a response in his brainwaves.

\section{Measurement and Feature Extraction}

The brainwave sensor used was Epoc+ (Emotiv Co., Ltd.,), which contains 14 electrodes. The sampling rate was $128 \mathrm{~Hz}$ and the frequency range was $0.2-45 \mathrm{~Hz}$.

The participants were seated in a chair in a relaxed position with their eyes closed and earplugs inserted to avoid stimuli other than the vibrational stimulus. Individual unperceivable vibration frequencies were presented to each participant. In addition, assuming the spoofing attack, the participants were given vibrations whose frequencies had been personalized for others.

Each measurement took 30 s. 10 personal vibration frequency measurements were taken, and 19 measurements using others' vibration frequencies were recorded, yielding a total of 29 measurements per participant. In the case of the personal vibration frequency, the participants were asked, after the measurement had been taken, whether they could perceive the vibration; if so the measured EEG was eliminated.

After all measurements had been taken, all EEGs were passed through a band pass filter with a passband of $4-43 \mathrm{~Hz}$. In addition, elimination of spike noises was performed. A statistical variance $\sigma$ was calculated from all amplitudes of an EEG; when the absolute value of each amplitude was larger than $\pm 3 \sigma$, it was replaced by a value of 0 .

It was impossible to extract individual features directly from the measured EEGs; therefore, the authors used fast Fourier transform (FFT) for their frequency analysis. The measured $30 \mathrm{~s}$ long EEGs were transformed by FFT to power spectra. Power-spectral elements were accumulated in $\theta(4-8 \mathrm{~Hz}), \alpha$ $(8-13 \mathrm{~Hz})$, and $\beta(13-43 \mathrm{~Hz})$ wavebands, and the ratio against 


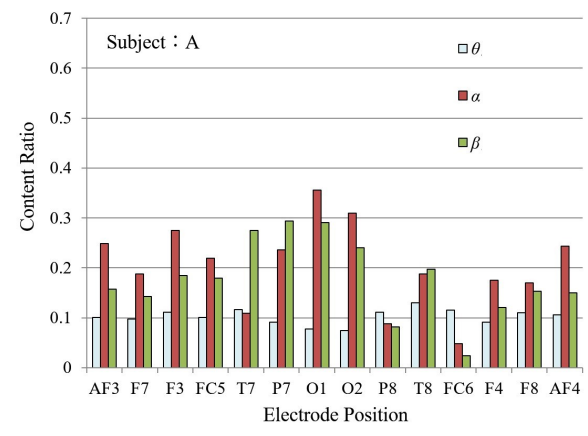

Fig. 3. Content ratios of a user when his vibration stimuli were presented.

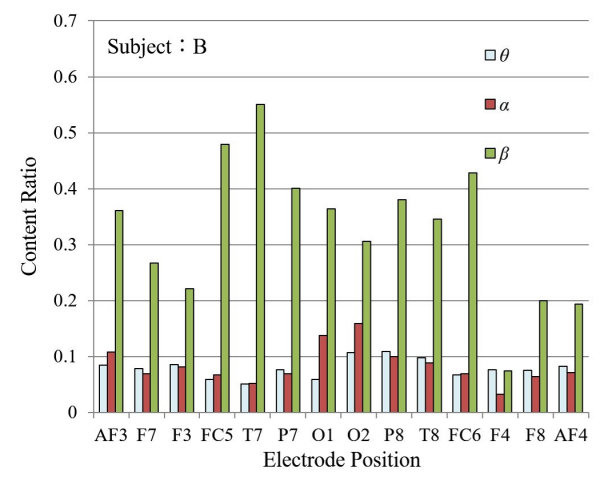

Fig. 4. Content ratios when the personal vibration stimulus was presented to other participants.

the total spectral power in all bands $(4-43 \mathrm{~Hz})$ was calculated in each band. In this paper, the ratio is called the content ratio and is used as an individual feature.

Examples are shown in Figs. 3 and 4; the names of the horizontal axes indicate the electrodes' positions. Figure 3 shows the content ratio of a participant when presented a stimulus with his vibration frequency, whereas in Fig. 4, the content ratios when vibration frequencies were applied to other participants are shown. These results show that the content ratios of a user are different from those of others when presented by his vibration. The content ratio is expected to be an individual feature.

\section{IV. $t$-TEST}

The authors evaluated the inducibility and individuality of the measured EEGs using a $t$-test, which is based on a simple assumption that if averages of two groups are equal, they are identical. A statistical probability of correctness of this assumption is obtained and called significance probability ( $p$ value). If the $p$-value is less than 0.05 , the assumption is incorrect and the two groups are regarded as not identical.

\section{A. Evaluation of Inducibility}

In order to examine whether unperceivable stimulation evoked responses in the brainwaves, the authors performed a "paired $t$-test" between a content ratio using unperceivable stimulation and one using perceivable stimulation of which vibration frequency was $160 \mathrm{~Hz}$. In addition, the authors used a paired $t$-test between a content ratio using unperceivable stimulation and one with no stimulation, which corresponds to the spontaneous brainwave case.

When two samples from an identical subject are compared, the paired $t$-test is defined by

$$
t=\frac{\bar{d}}{\sqrt{\frac{s^{2}}{n}},}
$$

where $n, \bar{d}$, and $s^{2}$ are the number of data, an average, and an unbiased variance, respectively.

The results of seven typical electrodes are summarized in Tables I and II. Equivalent results were also obtained in the remaining seven electrodes. Note that the number of participants was 10 and there were four measurements for each stimulation in this evaluation. The colored cells indicate that the $p$-value is less than 0.05 ; that is, the content ratio using unperceivable stimulation is not identical to that using perceivable stimulation in Table I and that in the spontaneous brainwave in Table II.

In these tables, the presence of many colored cells shows that the characteristics of EEGs obtained by presenting the unperceivable stimulation are different from those obtained when presenting perceivable stimulation and those in spontaneous brainwaves. This is an important evidence that the proposed stimulation method evoked some responses in brainwaves.

\section{B. Evaluation of Individuality}

In order to examine the differences in the participants' EEGs, the authors introduced "Welch's $t$-test", which is used when two samples are assumed to have different variances and is defined as

$$
t=\frac{\overline{x_{1}}-\overline{x_{2}}}{\sqrt{\frac{s_{1}^{2}}{n_{1}}+\frac{s_{2}^{2}}{n_{2}}}},
$$

where $n$ is the number of data, $\bar{x}$ indicates an average and $s^{2}$ is an unbiased variance in each sample.

In order to increase the number of data in this evaluation, each 30 s long EEG was divided into six sets of data, each 5 $\mathrm{s}$ long. As a result, $60(6 \times 10)$ data points from each subject and $114(6 \times 19)$ data points from other participants were used.

The results for seven typical electrodes in $\alpha$ waveband at each participant are summarized in Table III; the lack of space means that the results for other wavebands are omitted. The colored cells indicate that the $p$-value is less than 0.05 . The number of colored cells is relatively large and suggests that there is some difference between the evoked EEGs of an individual and those of others. This is an important evidence that the proposed personal stimulation results in different responses in brainwaves.

\section{Evaluation of Verification Performance}

The two previous sections confirmed the inducibility and individuality of EEGs evoked by unperceivable vibration stimulation. The authors then evaluated the verification performance using Euclidian distance matching. As an individual feature, 
TABLE I

Paired $t$-TeST WITH PERCEIVABle STIMUlation CASE.

\begin{tabular}{|c|c|c|c|c|c|c|c|}
\hline & O2 & P8 & T8 & FC6 & F4 & F8 & AF4 \\
\hline$\theta$ & 0.000116115 & 0.018171728 & $3.96598 \mathrm{E}-05$ & 0.085791653 & 0.02748563 & 0.002881972 & 0.105248499 \\
\hline$\alpha$ & 0.000135154 & 0.028332177 & 0.142009914 & 0.00126064 & 0.006781404 & 0.164354907 & 0.00155571 \\
\hline$\beta$ & 0.854710336 & 0.943197279 & 0.207847393 & 0.523600251 & $2.27773 \mathrm{E}-06$ & 0.007896605 & 0.00035038 \\
\hline
\end{tabular}

TABLE II

PAIRED $t$-TEST With NO STIMULATION CASE.

\begin{tabular}{|c|c|c|c|c|c|c|c|}
\hline & O2 & P8 & T8 & FC6 & F4 & F8 & AF4 \\
\hline$\theta$ & 0.001339109 & 0.379913701 & 0.003032733 & 0.032820719 & 0.009100676 & $5.57373 \mathrm{E}-05$ & 0.765502916 \\
\hline$\alpha$ & 0.277261843 & 0.00012984 & 0.062341879 & 0.003546791 & 0.644016704 & 0.102397597 & $4.05525 \mathrm{E}-05$ \\
\hline$\beta$ & 0.851542399 & 0.902483476 & 0.568804181 & 0.218893495 & $4.01216 \mathrm{E}-05$ & 0.009289228 & 0.18945805 \\
\hline
\end{tabular}

TABLE III

WELCH'S $t$-TEST IN $\alpha$ WAVE BAND AT EACH SUBJECT.

\begin{tabular}{|c|c|c|c|c|c|c|c|}
\hline & O2 & P8 & T8 & FC6 & F4 & F8 & AF4 \\
\hline A & 0.04313654 & 0.005929121 & 0.914753604 & 0.24464712 & $9.72585 \mathrm{E}-08$ & 0.03525443 & 0.836035142 \\
\hline B & $3.7979 \mathrm{E}-06$ & $2.36009 \mathrm{E}-12$ & $8.29146 \mathrm{E}-05$ & $1.75643 \mathrm{E}-07$ & $2.41027 \mathrm{E}-06$ & 0.001479384 & $8.93054 \mathrm{E}-12$ \\
\hline C & 0.139146031 & 0.000929386 & $1.52322 \mathrm{E}-07$ & 0.00276047 & 0.001774607 & 0.003093295 & 0.554951092 \\
\hline D & $4.68025 \mathrm{E}-30$ & $6.04791 \mathrm{E}-21$ & $6.81967 \mathrm{E}-14$ & $1.31049 \mathrm{E}-10$ & $1.21172 \mathrm{E}-06$ & $5.84115 \mathrm{E}-10$ & $8.91524 \mathrm{E}-15$ \\
\hline E & $2.62497 \mathrm{E}-05$ & 0.115712867 & 0.150996025 & 0.143598374 & 0.774849592 & 0.031660083 & 0.225427117 \\
\hline F & $2.46051 \mathrm{E}-05$ & 0.012901301 & 0.508118899 & 0.914554902 & 0.116354439 & 0.090233513 & 0.477710695 \\
\hline G & 0.01503026 & 0.009208582 & 0.004262535 & 0.821495846 & 0.079602058 & 0.006855861 & 0.210547351 \\
\hline H & 0.050349806 & 0.779079233 & 0.023548802 & 0.926504836 & 0.39132254 & 0.01251983 & 0.255361111 \\
\hline I & 0.875361068 & 0.170177621 & 0.86245459 & 0.024551227 & 0.012893132 & 0.004977405 & 0.701922777 \\
\hline J & $3.01354 \mathrm{E}-13$ & $1.5601 \mathrm{E}-14$ & $1.15063 \mathrm{E}-13$ & $2.41767 \mathrm{E}-12$ & $1.97732 \mathrm{E}-09$ & $9.25867 \mathrm{E}-13$ & $1.1003 \mathrm{E}-10$ \\
\hline K & $1.96239 \mathrm{E}-09$ & $6.67408 \mathrm{E}-06$ & $8.87611 \mathrm{E}-06$ & 0.011708553 & $2.00728 \mathrm{E}-05$ & $3.72185 \mathrm{E}-06$ & 0.000900477 \\
\hline L & 0.039785732 & 0.010381336 & 0.029708703 & 0.040473975 & 0.33755929 & 0.075596108 & 0.422731969 \\
\hline M & 0.021315513 & $5.03572 \mathrm{E}-06$ & 0.00132027 & 0.034519757 & 0.000559136 & 0.008309851 & $1.21458 \mathrm{E}-05$ \\
\hline N & $1.02926 \mathrm{E}-13$ & $8.35312 \mathrm{E}-11$ & $6.11707 \mathrm{E}-18$ & $2.36805 \mathrm{E}-09$ & $1.54387 \mathrm{E}-13$ & $6.01895 \mathrm{E}-13$ & $5.49329 \mathrm{E}-12$ \\
\hline O & 0.250240494 & 0.217436589 & 0.711383771 & 0.031871579 & 0.026662209 & 0.000998016 & 0.006764313 \\
\hline P & $9.46014 \mathrm{E}-06$ & 0.698370231 & 0.955380164 & 0.615764324 & 0.222010215 & 0.052390824 & 0.904411426 \\
\hline Q & $1.70152 \mathrm{E}-12$ & $6.392 \mathrm{E}-06$ & $6.08059 \mathrm{E}-11$ & 0.000453589 & $2.95629 \mathrm{E}-12$ & $5.71608 \mathrm{E}-07$ & $2.00724 \mathrm{E}-08$ \\
\hline R & $1.63145 \mathrm{E}-07$ & 0.000185345 & 0.000157364 & 0.147958294 & $1.78956 \mathrm{E}-08$ & 0.040178769 & 0.592313072 \\
\hline S & $2.14125 \mathrm{E}-08$ & $5.3548 \mathrm{E}-14$ & $1.86822 \mathrm{E}-07$ & $4.13381 \mathrm{E}-11$ & $1.94615 \mathrm{E}-09$ & 0.000501434 & $6.28423 \mathrm{E}-08$ \\
\hline T & 0.001318779 & 0.034203923 & 0.02932304 & 0.191338803 & $6.55561 \mathrm{E}-07$ & $1.22453 \mathrm{E}-06$ & $3.74379 \mathrm{E}-05$ \\
\hline
\end{tabular}

the content ratio of each brainwave band was used. However, to increase the number of dimensions of individual features, $\beta$ waveband was divided into lower $\beta(13-26 \mathrm{~Hz})$ and $\gamma(26-$ $43 \mathrm{~Hz}$ ), resulting in four dimensions. Five EEGs were used to make a template for each participant. The remaining EEGs were used in the test.

An equal error rate (EER), defined by a cross point where a false acceptance rate equaled a false rejection rate, was used as an evaluation index. The EERs at all electrodes are summarized in Table IV. There was no uniqueness in the

TABLE IV

EERS (\%) IN ALL ELECTRODES.

\begin{tabular}{|l|l|l|l|l|l|l|l|}
\hline AF3 & F7 & F3 & FC5 & T7 & P7 & O1 & O2 \\
\hline 32.5 & 31.1 & 34.0 & 33.0 & 36.9 & 34.6 & 37.6 & 35.0 \\
\hline P8 & T8 & FC6 & F4 & F8 & AF4 & Ave. & \\
\hline 31.9 & 33.8 & 34.0 & 33.8 & 32.2 & 31.5 & 33.7 & \\
\hline
\end{tabular}

EERs, and their average value was $33.7 \%$. This verification performance is unsatisfactory, but it was achieved using a simple feature (four content ratios) and Euclidian distance matching. It is expected that the verification performance will be improved by introducing other individual features and some learning-based verification methods.

\section{CONCLUSIONS}

In this study, the use of EEGs evoked by unperceivable vibration stimulation was proposed. EEGs in 20 participants were measured. The content ratios of the power spectra in $\theta, \alpha$, and $\beta$ wavebands were used as an individual feature, and their inducibilities and individualities were examined and confirmed using $t$-test. Finally, the verification performance using the content ratio in evoked EEGs as an individual feature was examined using Euclidian distance matching. As a result, an average EER of all electrodes was approximately $34 \%$. This indicates the feasibility of person authentication using unperceivable vibration stimulation. This study has paved the way for research into other aspects related to the proposed method. For example, new individual features and learningbased verification methods could be introduced, features or results from some electrodes could be combined, and the number of research participants could be increased.

\section{REFERENCES}

[1] M.D. Pozo-Banos, J.B. Alonso, J.R. Ticay-Rivas, and C.M. Travieso, "Electroencephalogram subject identification: A review," Expert Systems with Applications, pp. 6537-6554, 2014.

[2] I. Nakanishi and M. Hattori, "Biometric potential of brain waves evoked by invisible visual stimulation," Proc. of 2017 International Conference on Biometrics and Kansei Engineering (ICBAKE2017), pp. 94-99, 2017.

[3] T. Maruoka, K. Kambe, H. Harada, and I. Nakanishi, "A Study on evoked potential by inaudible auditory stimulation toward continuous biometric authentication," Proc. of 2017 IEEE R10 Conference (TENCON2017), pp. 1171-1174, 2017.

[4] G.K. Singhal, and P. Ramkumar, "Person identification using evoked potentials and peak matching," Proc. of 2007 Biometric Symposium, 2007.

[5] S. J. Bolanowski, G. A. Gascheider, R. T. Verrillo, and C. M. Checkosky, "Four channels mediate the mechanical aspects of touch," Journal of the Acoustical Society of America, pp. 1680-1694, 1988.

[6] M. Tada, "Individual difference in contact," Journal of the Robotics Society of Japan (in Japanese), pp. 469-471, 2012. 